\title{
Adenosine Triphosphate Exposes Dormant Pulmonary Vein Conduction Responsible for Recurrent Atrial Tachyarrhythmias Importance of Evaluating the Dormant Conduction During the Re-Do Ablation Procedure
}

\author{
Shinsuke Miyazaki, MD*; Taishi Kuwahara, MD*; Atsushi Kobori, MD*; \\ Yoshihide Takahashi, MD*; Asumi Takei, MD*; Akira Sato, MD*; \\ Atsushi Takahashi, MD*; Mitsuaki Isobe, MD**
}

\begin{abstract}
A 61-year-old man suffered from atrial tachycardia (AT) following pulmonary vein isolation (PVI) treatment of atrial fibrillation. During a re-do procedure, all pulmonary veins (PVs) were isolated from the left atrium (LA) and programmed stimulation could not induce any atrial arrhythmias. Adenosine triphosphate exposed dormant PV-LA conduction at the right superior PV. PV firing during the reconnection caused AT with the same P-wave morphology as clinical AT. For 6 months after the elimination of that dormant PV conduction, the patient has been free of any AT. Evaluation of dormant PV conduction was effective in curing the recurrent AT after PVI. (Circ J 2009; 73: 1160-1162)
\end{abstract}

Key Words: AF ablation; ATP; Dormant conduction

D ormant pulmonary vein $(\mathrm{PV})$ conduction can be revealed by the administration of adenosine triphosphate (ATP) just after PV isolation (PVI)! Recent studies have demonstrated that radiofrequency (RF) applications delivered to eliminate any ATP-induced dormant PV conduction have reduced the clinical recurrence of atrial fibrillation (AF) after initial PVI, 3 However, the usefulness of ATP administration during a re-do procedure for recurrent atrial arrhythmias has not been elucidated. We present a case in which ATP was effective in identifying the dormant PV conduction responsible for a recurrent atrial tachyarrhythmia.

\section{Case Report}

A 61-year-old man with a history of drug-resistant paroxysmal AF had undergone extensive PVI. The left atrial diameter and left atrial ejection fraction were within normal limits. During the initial procedure, double circular mapping catheters (Lasso, Biosense Webster) were placed in the superior and inferior PVs, and the left-sided and right-sided ipsilateral PVs were circumferentially and extensively ablated, respectively, under fluoroscopic and electrophysiologic guidance, ${ }^{4} 5$ After isolating all the PVs, no reconnec-

(Received May 12, 2008; revised manuscript received June 27, 2008; accepted July 14, 2008; released online December 19, 2008)

*Cardiovascular Center, Yokosuka Kyosai Hospital, Yokosuka, **Department of Cardiovascular Medicine, Tokyo Medical and Dental University, Tokyo, Japan

Mailing address: Shinsuke Miyazaki, MD, Cardiovascular Center, Yokosuka Kyosai Hospital, 1-16 Yonegahamadori, Yokosuka 2388558, Japan. E-mail: mshinsuke@k3.dion.ne.jp

All rights are reserved to the Japanese Circulation Society. For permis-

sions, please e-mail: cj@j-circ.or.jp tion between the left atrium (LA) and the PVs was observed during the administration of $40 \mathrm{mg}(0.8 \mathrm{mg} / \mathrm{kg})$ of ATP under an isoproterenol (ISP) infusion $(2 \mu \mathrm{g} / \mathrm{min})$. However, 4 weeks after the procedure, he began to suffer from recurrent atrial tachycardia (AT) (Figure 1), which was sustained for several hours, and occurred 2-3 times per week.

In the re-do ablation session, the patient presented in sinus rhythm. All 4 PVs were isolated, and dissociated PV potentials were observed in the right superior PV (RSPV) (Figure 2A). An ISP infusion $(2 \mu \mathrm{g} / \mathrm{min})$ could not induce any PV reconnections, and no AT could be provoked by programmed stimulation.

We then administered $40 \mathrm{mg}$ of ATP to evaluate the existence of any dormant PV conduction. A transient LAPV reconnection reproducibly appeared at the RSPV (Figure 2B). Rapid PV firing occurred spontaneously from the RSPV during the reconnection, which caused an AT with the same P-wave morphology as the clinical AT (Figures 3A,B). We eliminated the RSPV-LA conduction with a single RF application while the reconnection was exposed (Figures 4B,C). After the RF application, the RSPV-LA reconduction no longer appeared and after followup of 6 months, the patient has been free of any atrial arrhythmias without any antiarrhythmic drugs.

\section{Discussion}

Previous studies have identified the PVs as the source of triggers initiating and perpetuating AF. PVI is now an established therapy for curing AF, 6 ,7 Arentz et al reported that adenosine induced transient conduction in $25 \%$ of PVs immediately after successful isolation! In that study, the dose of the adenosine was $12-18 \mathrm{mg}$, and the adenosineinduced PV reactivation persisted for approximately 20 s. 
A

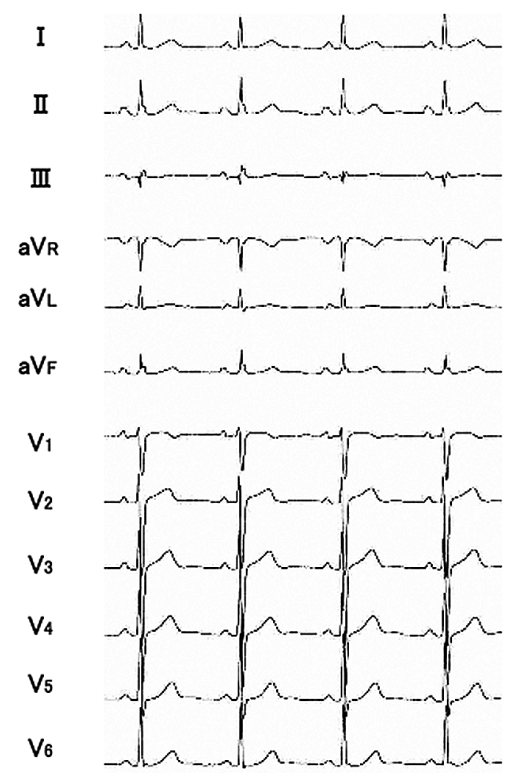

B

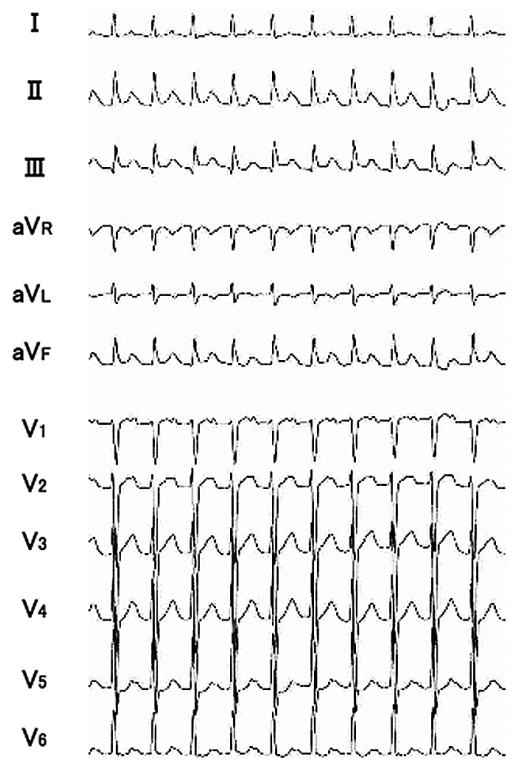

Figure 1. Twelve-lead ECG during sinus rhythm (A) and recurrent clinical atrial tachycardia (B).
A

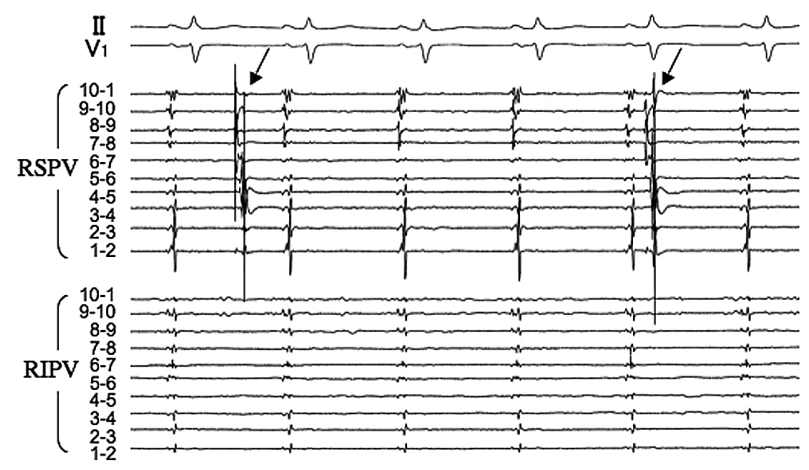

B

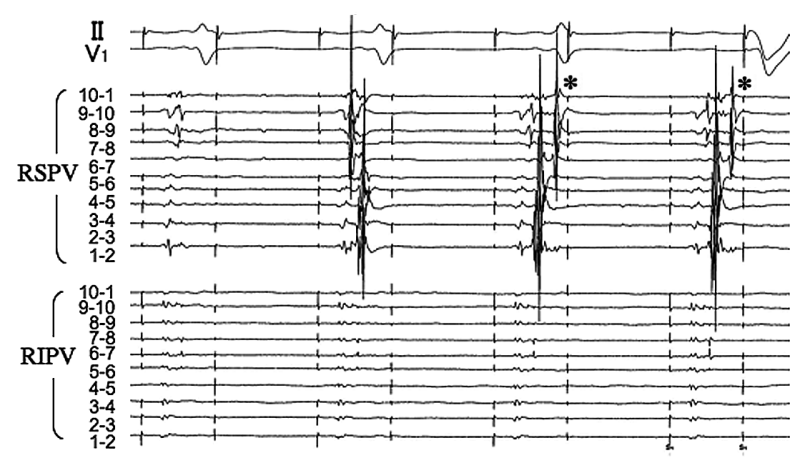

Figure 2. Double circular mapping catheters placed in the right superior and inferior pulmonary veins (RSPV and RIPV). Dissociated pulmonary vein spikes (arrows) can be seen in the RSPV (A). A left atrium-RSPV transient reconnection $(*)$ appeared after intravenous injection of adenosine triphosphate during atrial and ventricular sequential pacing $(\mathbf{B})$.

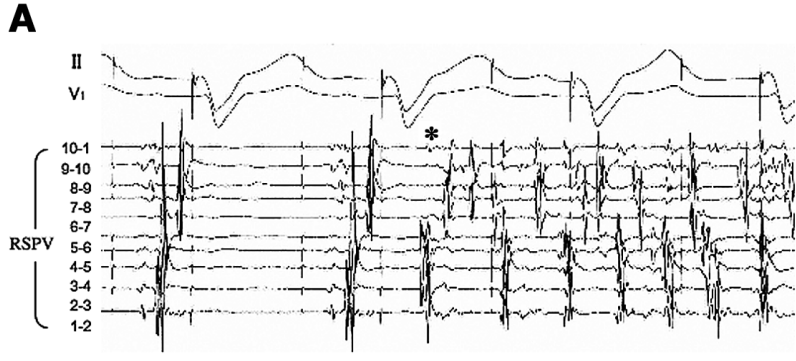

B
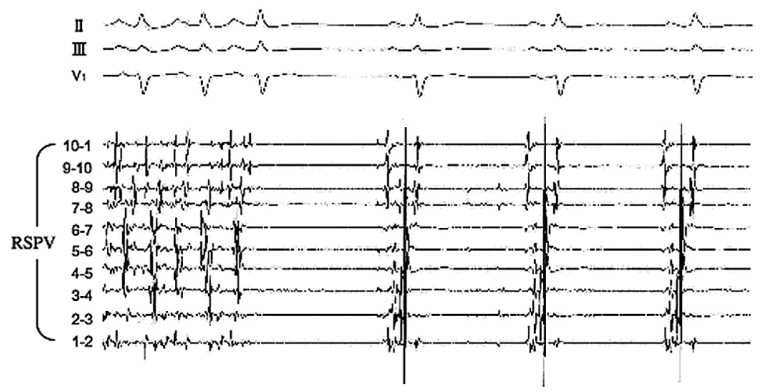

Figure 3. After the electrical reconnection between the pulmonary vein (PV) and left atrium appeared, rapid firing within the PV was induced (A), and the PV firing then caused an atrial tachycardia, which was terminated at the same time the PV firing was terminated (B). RSPV, right superior PV.

Furthermore, it has been shown that additional RF applications after the PVI in order to eliminate any ATP-induced dormant PV conduction improved the clinical outcome of the initial PVI procedure, 23 In those studies, the dose of the ATP was $20-30 \mathrm{mg}$ and it was administered during intravenous ISP infusion. Although the reason the ATP was able to elucidate the dormant conduction was not sufficiently clarified, the following mechanism was suggested: adenosine activates the outward potassium current via purinergic A1-receptors. The resulting hyperpolarization of the cell 
A

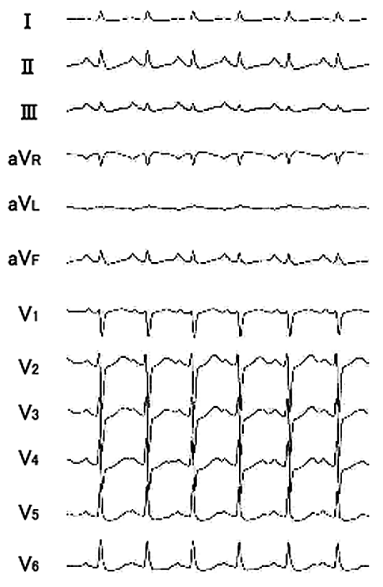

B

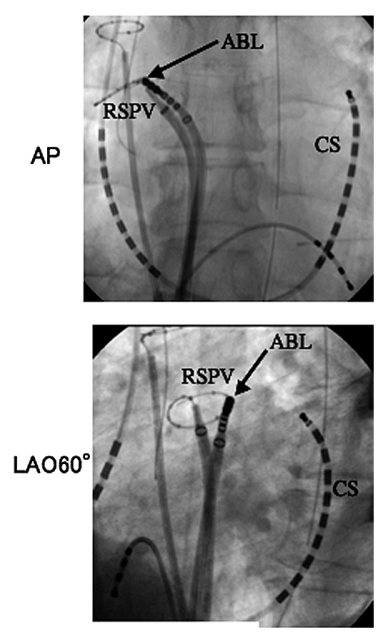

C

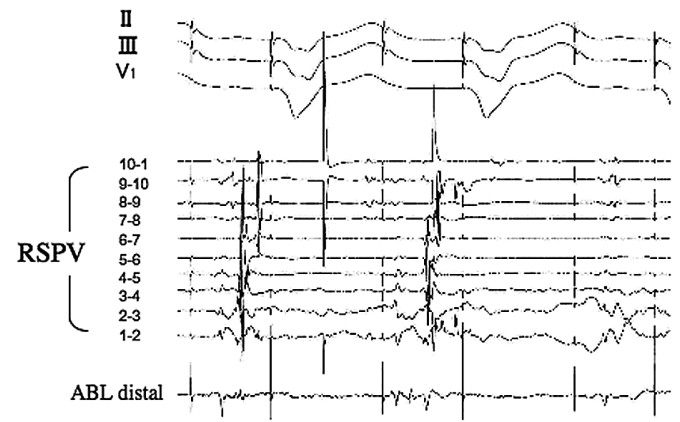

Figure 4. Atrial tachycardia (AT) during pulmonary vein (PV) firing has the same $\mathrm{P}$-wave morphology (A) as the clinical AT. One radiofrequency (RF) energy application at the superior-posterior ostium of the right superior PV (RSPV) (B) eliminated the dormant PV conduction provoked by adenosine triphosphate (ATP) injection (C). Following RF application, the left atrium-PV reconnection could no longer be induced by ATP injection. ABL, ablation catheter; CS, coronary sinus.

membrane and shortening of the action potential/refractory period may facilitate electrotonic conduction.

Given that the dormant conduction was eliminated by a single RF application in the present case, the induced AT was likely to have occurred because of electrical conduction of the PV firing to the LA through a conduction gap. Because the $\mathrm{P}$ waves of the clinical AT had the same morphology as those of the induced AT, the clinical AT may have been caused by a similar mechanism. The fact that no further frequent clinical AT has occurred since the re-do session strongly suggests that the dormant PV conduction was responsible for the occurrence of the clinical condition. Actually, we need to rule out a focal AT, a micro- or a macro-reentrant tachycardia, including the ablation site, as a critical lesion for maintaining those tachycardias; however, considering that the AT was only induced after rapid PV firing following the LA-PV re-connection, the aforementioned mechanism is more likely. The reason for the difference in the persistence of the clinical and induced ATs remains uncertain. Some neurohormonal conditions, such as increased sympathetic nervous activity, may have been associated with the long duration of the clinical AT.

\section{Clinical Implications}

These observations confirm the importance of dormant PV conduction as a cause of recurrent atrial arrhythmias following AF ablation. When no PV-LA conduction is observed during a re-do ablation session, evaluation of the existence of any dormant PV conduction using ATP may useful for clarifying the etiology and cure of a recurrent atrial arrhythmia, in addition to that done in the initial session during the acute phase.

\section{References}

1. Arentz T, Macle L, Kalusche D, Hocini M, Jais P, Shah D, et al. 'Dormant' pulmonary vein conduction revealed by adenosine after ostial radiofrequency catheter ablation. $J$ Cardiovasc Electrophysiol 2004; 15: 1041-1047.

2. Hachiya H, Hirao K, Takahashi A, Nagata Y, Suzuki K, Maeda S, et al. Clinical implication of reconnection between the left atrium and isolated pulmonary vein provoked by adenosine triphosphate after extensive encircling pulmonary vein isolation. J Cardiovasc Electrophysiol 2007; 18: $1-7$.

3. Matsuo S, Yamane T, Date T, Inada K, Kanzaki S, Tokuda M, et al. Reduction of AF recurrence after pulmonary vein isolation by eliminating ATP-induced transient venous re-conduction. $J$ Cardiovasc Electrophysiol 2007; 18: 704-708.

4. Kuwahara T, Takahashi A, Kobori A, Miyazaki S, Takahashi Y, Takei A, et al. Safe and effective ablation of atrial fibrillation: Importance of esophageal temperature monitoring to avoid periesophageal nerve injury as a complication of pulmonary vein isolation. $J$ Cardiovasc Electrophysiol 2009; 20: 1-6.

5. Miyazaki S, Kuwahara T, Takahashi A, Kobori A, Takahashi Y, Toshihiro N, et al. Effect of left atrial ablation on the quality of life in patients with atrial fibrillation. Circ J 2008; 72: 582-587.

6. Haissaguerre M, Jais P, Shah DC, Takahashi A, Hocini M, Quiniou $\mathrm{G}$, et al. Spontaneous initiation of atrial fibrillation by ectopic beats originating from the pulmonary veins. N Engl J Med 1998; 339: 659666.

7. Haissaguerre M, Shah DC, Jais P, Hocini M, Yamane T, Deisenhofer I, et al. Electrophysiological breakthroughs from the left atrium to the pulmonary veins. Circulation 2000; 102: 2463-2465. 